9. Paul, C. P., Good, P. D., Winer, I. \& Engelke, D. R. Effective expression of small interfering RNA in human cells. Nature Biotechnol. 20, 505-508 (2002).

10. Lois, C., Hong, E. J., Pease, S., Brown, E. J. \& Baltimore, D. Germline transmission and tissue-specific expression of transgenes delivered by lentiviral vectors. Science 295, 868-872 (2002).

11. Birrell, G. W., Giaever, G., Chu, A. M., Davis, R. W. \& Brown, J. M. A genome-wide screen in Saccharomyces cerevisiae for genes affecting UV radiation sensitivity. Proc. Natl Acad. Sci. USA 98, 12608-12613 (2001)

12. Giaever, G. et al. Functional profiling of the Saccharomyces cerevisiae genome. Nature 418, 387-391 (2002).

13. Winzeler, E. A. et al. Functional characterization of the S. cerevisiae genome by gene deletion and parallel analysis. Science 285, 901-906 (1999)

14. Ghoda, L., Sidney, D., Macrae, M. \& Coffino, P. Structural elements of ornithine decarboxylase required for intracellular degradation and polyamine-dependent regulation. Mol. Cell. Biol. 12, 2178-2185 (1992)

15. Chen, P. \& Hochstrasser, M. Autocatalytic subunit processing couples active site formation in the $20 \mathrm{~S}$ proteasome to completion of assembly. Cell 86, 961-972 (1996)

16. Heinemeyer, W., Fischer, M., Krimmer, T., Stachon, U. \& Wolf, D. H. The active sites of the eukaryotic $20 S$ proteasome and their involvement in subunit precursor processing. J. Biol. Chem. 272, 25200-25209 (1997).

17. Bochtler, M., Ditzel, L., Groll, M., Hartmann, C. \& Huber, R. The proteasome. Annu. Rev. Biophys. Biomol. Struct. 28, 295-317 (1999).

18. Coux, O. An interaction map of proteasome subunits. Biochem. Soc. Trans. 31, 465-469 (2003).

19. Kim, S. Y., Herbst, A., Tworkowski, K. A., Salghetti, S. E. \& Tansey, W. P. Skp2 regulates Myc protein stability and activity. Mol. Cell 11, 1177-1188 (2003)

Supplementary Information accompanies the paper on www.nature.com/nature.

Acknowledgements We thank T. Moore and B. Simmons from Open Biosystems for their help in organizing and rearraying the library, and colleagues at CSHL and elsewhere (as indicated in Supplementary Table 1) as well as J. LaBaer and C. Perou for curating gene lists. G. Katari and J. Faith helped with bioinformatic analysis and shRNA choice, and members of the Lowe laboratory (CSHL) provided advice on vector optimization. This work was supported by an Innovator Award from the US Army Breast Cancer Research Program (G.J.H.), a contract from the National Cancer Institute (G.J.H.), grants from the NIH (G.J.H., W.R.M., S.J.E.) and the US Army Breast Cancer Research Program (G.J.H., D.S.C.), the Howard Hughes Medical Institute (S.J.E.), and by generous support from Oncogene Sciences and Merck. P.J.P. is an Arnold and Mabel Beckman Fellow of the Watson School of Biological Sciences and is supported by a predoctoral fellowship from the US Army Breast Cancer Research Program. J.M.S. is supported by a postdoctoral fellowship from the US Army Prostate Cancer Research Program. S.J.E. is an Investigator of the Howard Hughes Medical Institute. G.J.H. is a Rita Allen Foundation Fellow.

Competing interests statement The authors declare that they have no competing financial interests.

Correspondence and requests for materials should be addressed to G.J.H. (hannon@cshl.org) or S.J.E. (selledge@genetics.med.harvard.edu).

\section{A large-scale RNAi screen in human cells identifies new components of the p53 pathway}

\author{
Katrien Berns $^{1 *}$, E. Marielle Hijmans ${ }^{1 *}$, Jasper Mullenders ${ }^{1}$, \\ Thijn R. Brummelkamp ${ }^{1}$, Arno Velds ${ }^{1}$, Mike Heimerikx ${ }^{1}$, \\ Ron M. Kerkhoven ${ }^{1}$, Mandy Madiredjo ${ }^{1}$, Wouter Nijkamp ${ }^{1}$, \\ Britta Weigelt ${ }^{2}$, Reuven Agami ${ }^{3}$, Wei Ge ${ }^{4}$, Guy Cavet ${ }^{4}$, Peter S. Linsley ${ }^{4}$, \\ Roderick L. Beijersbergen ${ }^{1} \&$ René Bernards
}

${ }^{1}$ Division of Molecular Carcinogenesis and Center for Biomedical Genetics, ${ }^{2}$ Division of Experimental Therapy, and ${ }^{3}$ Division of Tumor Biology, The Netherlands Cancer Institute, Plesmanlaan 121, 1066 CX Amsterdam, The Netherlands

${ }^{4}$ Rosetta Inpharmatics, Inc., 12040 115th Avenue NE, Kirkland, Washington 98034, USA

* These authors contributed equally to this work

RNA interference (RNAi) is a powerful new tool with which to perform loss-of-function genetic screens in lower organisms and can greatly facilitate the identification of components of cellular signalling pathways ${ }^{1-3}$. In mammalian cells, such screens have been hampered by a lack of suitable tools that can be used on a large scale. We and others have recently developed expression vectors to direct the synthesis of short hairpin RNAs (shRNAs) that act as short interfering RNA (siRNA)-like molecules to stably suppress gene expression ${ }^{4,5}$. Here we report the construction of a set of retroviral vectors encoding 23,742 distinct shRNAs, which target 7,914 different human genes for suppression. We use this RNAi library in human cells to identify one known and five new modulators of $p 53$-dependent proliferation arrest. Suppression of these genes confers resistance to both $p 53$-dependent and $p 19^{A R F}$-dependent proliferation arrest, and abolishes a DNAdamage-induced G1 cell-cycle arrest. Furthermore, we describe siRNA bar-code screens to rapidly identify individual siRNA vectors associated with a specific phenotype. These new tools will greatly facilitate large-scale loss-of-function genetic screens in mammalian cells.

RNAi is a defence mechanism triggered by double-stranded (ds)RNAs to protect cells from parasitic nucleic acids. The dsRNAs are processed into siRNAs, which target homologous RNAs for destruction ${ }^{6}$. In mammalian cells, an RNAi response can be triggered by 21-base-pair siRNAs, which can cause strong, but transient, inhibition of gene expression ${ }^{7}$. By contrast, vector-expressed shRNAs can suppress gene expression over prolonged periods ${ }^{4,5}$. In the current study, we create a large set of vectors and use them to search for components of the p53 tumour-suppressor pathway. This pathway is crucial for genome integrity as it transmits both antiproliferative and pro-apoptotic signals in response to a variety of stress signals ${ }^{8}$.

To construct a human RNAi library (the 'NKi library'), we selected 7,914 human genes for shRNA-mediated reduction in expression, known as knockdown. This collection of genes includes components of major cellular pathways, including the cell cycle, transcription regulation, stress signalling, signal transduction and important biological processes such as biosynthesis, proteolysis and metabolism. In addition, genes implicated in cancer and other diseases are included in the library (see Supplementary Table 1). To increase the likelihood of obtaining a significant inhibition of gene expression, we constructed three different shRNA vectors against each gene $(23,742$ vectors in total; Fig. 1a and Supplementary Fig. 1). The oligonucleotides specifying the shRNAs were annealed and cloned in a high-throughput fashion into pRetroSuper (pRS), a retroviral vector that contains the shRNA expression cassette ${ }^{9}$. Using a pool of three knockdown vectors against a single gene, we obtain on average $70 \%$ inhibition of expression for approximately $70 \%$ of the genes in the library (see ref. 10; data not shown). The vector-based shRNA library can be used for functional genetic screens in both short-term and long-term assays using DNA transfection or retroviral transduction.

To validate the RNAi library, we developed a cell system to screen for bypass of p53-dependent proliferation arrest. We generated primary human BJ fibroblasts, which ectopically express the murine ecotropic receptor, the telomerase catalytic subunit (TERT) and a temperature-sensitive allele of SV40 large T antigen (tsLT), yielding BJ-TERT-tsLT cells. As can be seen in Fig. 1b, these cells proliferate when grown at $32{ }^{\circ} \mathrm{C}$, the temperature at which tsLT binds and inactivates both retinoblastoma protein $(\mathrm{pRB})$ and $\mathrm{p} 53$, but enter into a synchronous proliferation arrest after a shift to $39^{\circ} \mathrm{C}$, at which tsLT is inactive. To determine whether this proliferation arrest is p53 dependent, we infected BJ-TERT-tsLT cells with pRS-p53 (which targets $p 53$ for suppression) at $32^{\circ} \mathrm{C}$, and shifted them to $39^{\circ} \mathrm{C}$ after two days. Figure $1 \mathrm{~b}$ shows that knockdown of p53 allowed temperature-shift-induced proliferation arrest to be bypassed. Knockdown of the RB pathway component $p 16^{I N K 4 A}$ alone did not allow growth arrest to be bypassed, but simultaneous suppression of both $p 16^{I N K 4 A}$ and $p 53$ yielded a further increase in escape from growth arrest compared with knockdown of $p 53$ alone (Fig. 1b). Thus, the conditional proliferation arrest in BJ fibroblasts depends primarily on $p 53$.

We isolated polyclonal plasmid DNA from each of the 83 pools of 
NKi RNAi library vectors (Fig. 1a), and transfected this DNA into a packaging cell line to generate high-titre retroviral supernatants. These retroviruses were then used to infect BJ-TERT-tsLT cells at $32^{\circ} \mathrm{C}$. We co-infected cells with a shRNA vector that targets p1 ${ }^{\text {INK4A }}$, because suppression of this gene further enhances colony formation induced by $p 53$ knockdown (Fig. 1b). After two days, the cells were shifted to $39^{\circ} \mathrm{C}$ and monitored for the appearance of proliferating colonies. In first-round screening, we identified six pools of infected cells that form colonies at $39^{\circ} \mathrm{C}$. To detect the biologically active shRNAs in these vector pools, we isolated several individual colonies of proliferating BJ fibroblasts and recovered the shRNA inserts of the vectors by polymerase chain reaction (PCR; see Methods). These shRNA inserts were then re-cloned, and their identity was established by DNA sequence analysis.

The majority of colonies of BJ fibroblasts that proliferated at $39^{\circ} \mathrm{C}$ contained multiple shRNA inserts. Only those shRNA inserts that were present in multiple independently derived colonies were analysed in second-round selection in BJ-TERT-tsLT fibroblasts. Using this approach, we identified shRNAs against six genes that could suppress the temperature-shift-induced proliferation arrest in the BJ-TERT-tsLT cells in the second round. One of the six genes was p53 itself, which underscores the quality of the NKi library. In addition, we found that individual shRNAs against RPS6KA6 (ribosomal S6 kinase 4, RSK4), HTATIP (histone acetyl transferase TIP60), HDAC4 (histone deacetylase 4), KIAA0828 (a putative $S$-adenosyl-L-homocysteine hydrolase, SAH3) and CCT2 (Tcomplex protein $1, \beta$-subunit) prevented the p53-dependent growth arrest in the BJ-TERT-tsLT fibroblasts (Fig. 1c). Knockdown of all five newly identified genes also mediated escape from proliferation arrest in BJ-TERT-tsLT cells when tested in the absence of $p 16^{I N K 4 A}$ knockdown vector (Fig. $2 \mathrm{~b}$ ), suggesting that inhibition of these genes primarily allows the bypass of the p53 response (see below).

Some siRNAs have 'off-target' effects, which are often the result of partial homology to other transcripts ${ }^{11,12}$. The shRNA library was designed to avoid off-target effects by minimizing homology of shRNAs to other transcripts (see Methods). Furthermore, it is very unlikely that two independent siRNAs against the same transcript target a common off-target transcript for suppression. For three of the five newly identified genes, we found that two of the three shRNAs present in the library mediated escape from growth arrest in the BJ-TERT-tsLT cells, suggesting that the effects of the shRNAs were 'on-target'. For the two genes for which only one functional shRNA from the library was found, we designed a second active

a

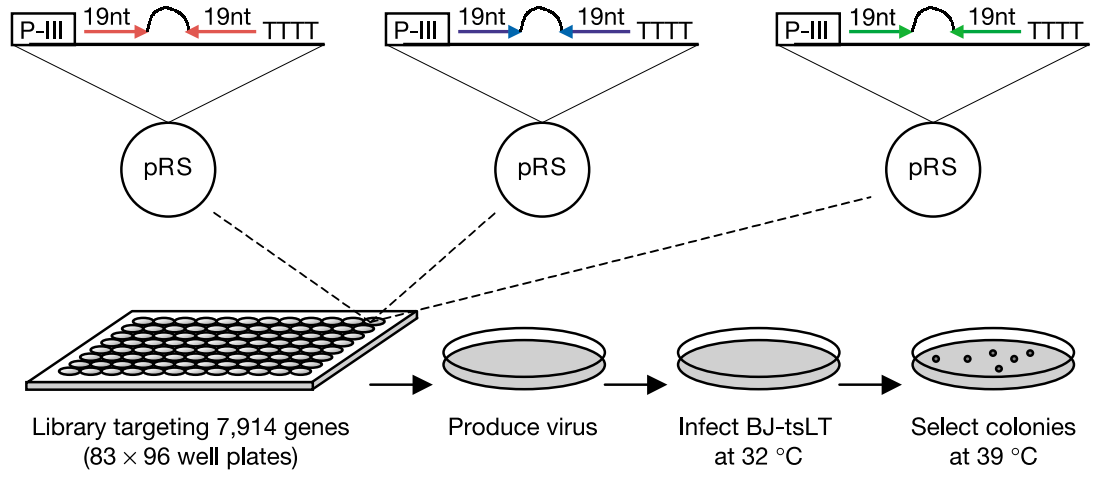

b

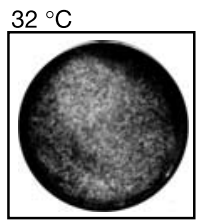

Control

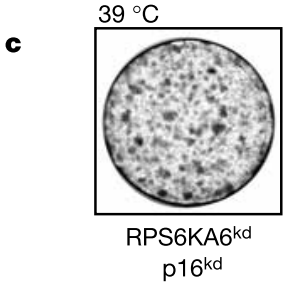

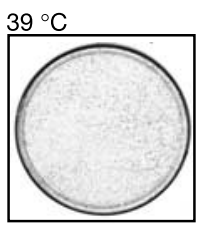

Control

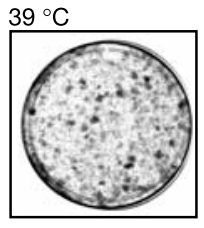

HTATIPkd p16kd

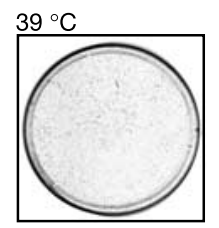

p16kd

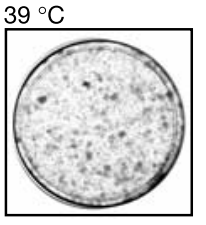

HDAC4kd p16 ${ }^{\mathrm{kd}}$

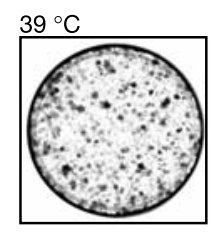

$\mathrm{p} 53^{\mathrm{kd}}$

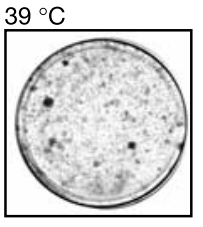

CCT2 ${ }^{\mathrm{kd}}$

$\mathrm{p} 16^{\mathrm{kd}}$

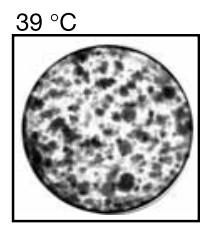

$\mathrm{p} 53^{\mathrm{kd}} / \mathrm{p} 16^{\mathrm{kd}}$

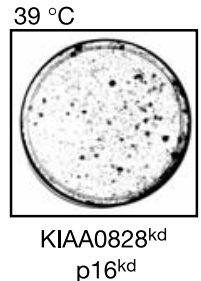

Figure 1 RNAi library screen in conditionally immortalized cells. a, Construction of the NKi RNAi library. From each gene transcript, three 19-nucleotide (nt) sequences were designed, and hairpin derivatives (59-mer oligonucleotides; see Supplementary Fig. 1) were cloned into pRS. Three vectors targeting one gene were combined in a single well of a 96-well plate. From each 96-well plate, DNA was pooled, and high-titre polyclonal virus was produced and used to infect BJ-tsLT cells at $32^{\circ} \mathrm{C}$. After two days, cells were shifted to $39^{\circ} \mathrm{C}$, and after three weeks colonies were isolated and analysed. $\mathbf{b}$, The growth arrest of temperature-shifted BJ-tsLT cells is p53 dependent. Co-infection with an shRNA against $p 16^{I N K 4 A}$ enhanced colony outgrowth. Cells were infected with the indicated shRNA knockdown $(\mathrm{kd})$ vectors at $32^{\circ} \mathrm{C}$. Fifty thousand cells were seeded at both $32^{\circ} \mathrm{C}$ and $39^{\circ} \mathrm{C}$, and colonies were stained after three weeks. c, Knockdown of the five newly identified genes allows bypass of the p53-dependent growth arrest in BJ-tsLT cells. 
shRNA vector targeting the same transcript (see Supplementary Table 2). Figure 2 a shows that transient co-transfection of each of the five pairs of two active shRNA vectors with the corresponding full-length complementary DNA expression vectors resulted in a substantial suppression of their cognate gene. Furthermore, we also observed a significant suppression of the corresponding endogen- ous transcripts (see Supplementary Fig. 2). Moreover, all five pairs of two active shRNA vectors were able to rescue the temperatureshift-induced growth arrest in the BJ-TERT-tsLT cells (Fig. 2b). Together, these data suggest that the observed escape from growth arrest is due to on-target effects of the shRNA vectors on the corresponding genes. a
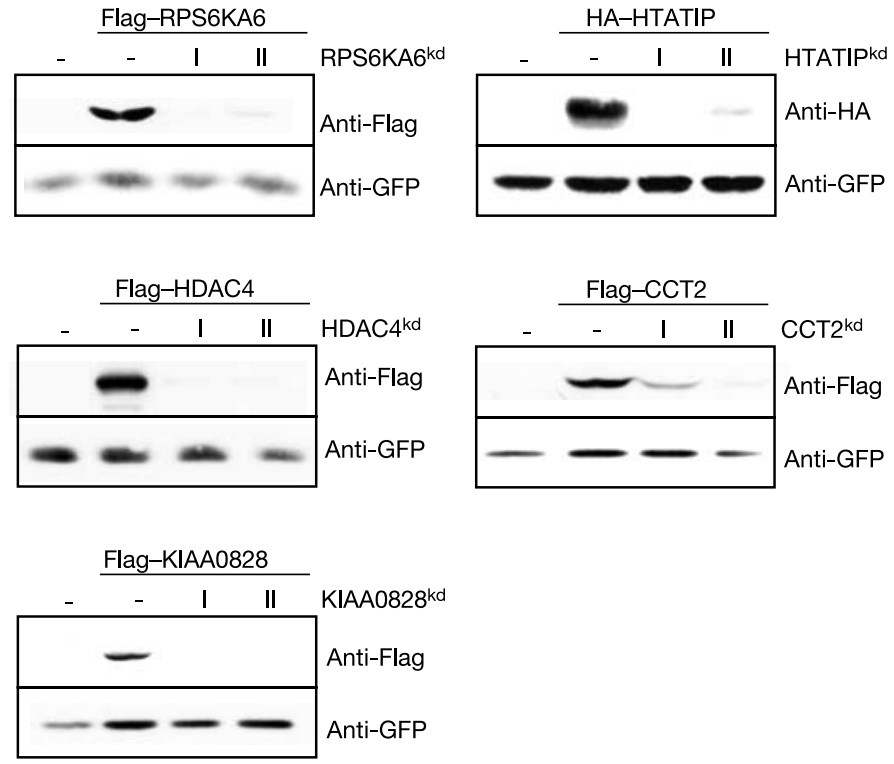

b

\section{BJ / tsLT}

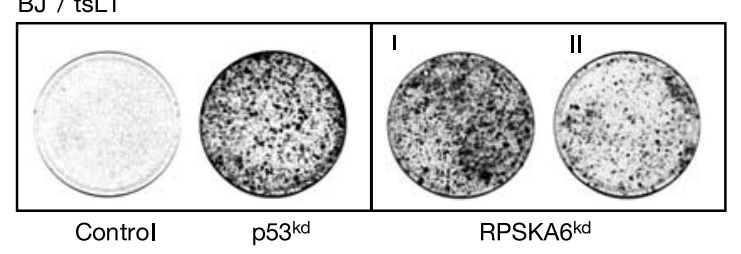

HTATIPkd

$\mathrm{HDAC}^{\mathrm{kd}}$

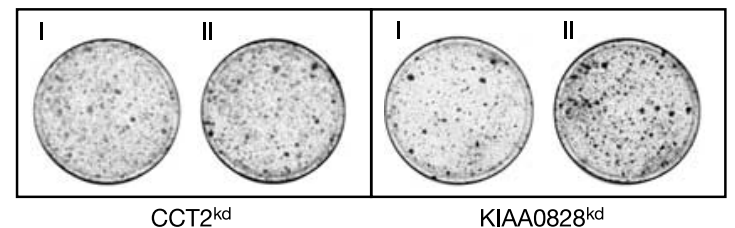

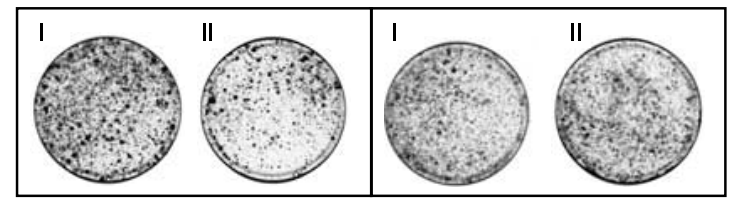
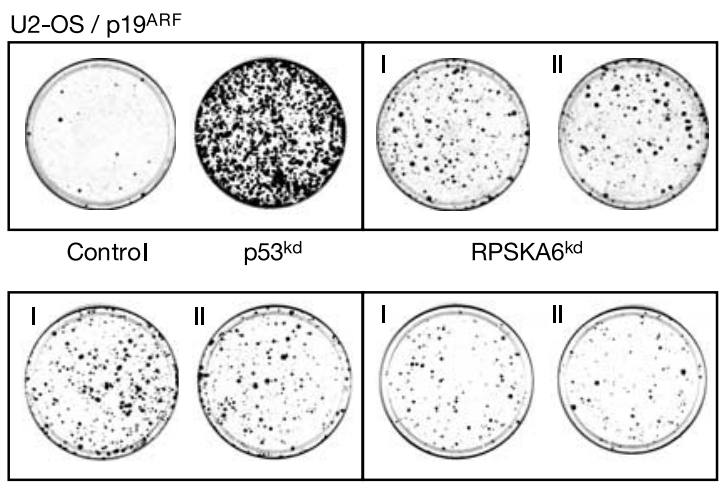

HTATIPk

$\mathrm{HDAC}^{\mathrm{kd}}$

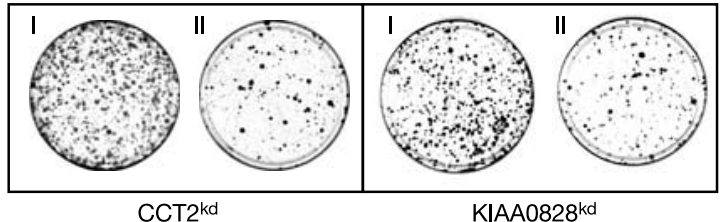

d

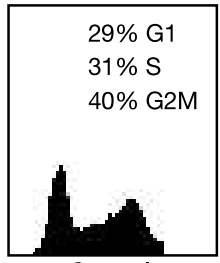

Control

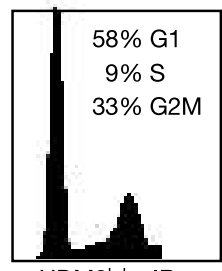

$\mathrm{HDM}^{\mathrm{kd}}+\mathrm{IR}$

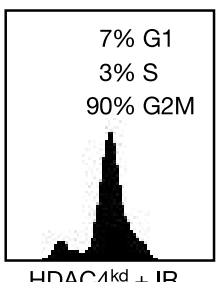

HDAC4 $^{\mathrm{kd}}+\mathrm{IR}$

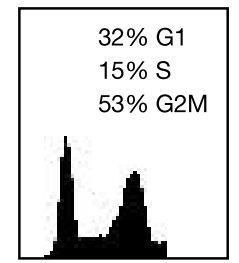

Control + IR

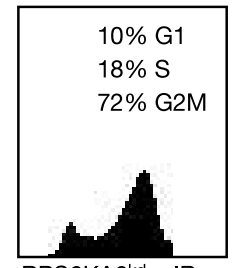

RPS6KA6 $6^{\mathrm{kd}}+\mathrm{IR}$

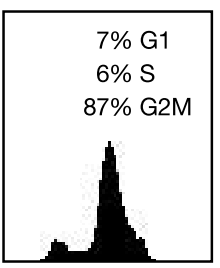

$\mathrm{CCT} 2^{\mathrm{kd}}+\mathrm{IR}$

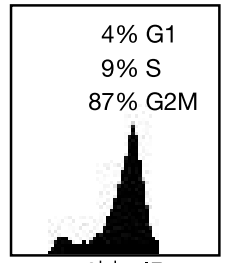

$\mathrm{p} 53^{\mathrm{kd}}+\mathrm{IR}$

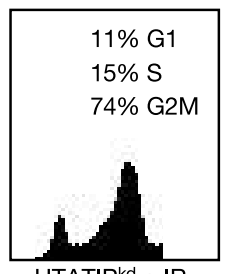

HTATIP $^{k d}+$ IR

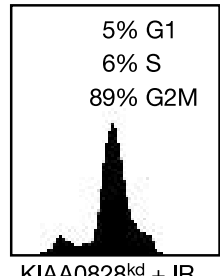

Figure 2 Validation of new p53 pathway components. a, Knockdown of newly identified p53 pathway components. U2-OS cells were co-transfected with the shRNA vectors (I or II) and their corresponding tagged (HA or Flag) cDNA expression vectors as indicated. Extracts were immunoblotted against Flag, HA or GFP (control). b, Knockdown of the five pairs of shRNA vectors rescue growth arrest in BJ-TERT-tsLT cells. Cells were infected with the indicated shRNA vectors (in the absence of the $p 16^{\text {INK4A }}$ knockdown vector), selected at $39^{\circ} \mathrm{C}$, and colonies were stained after three weeks. c, Two independent
shRNA vectors of each indicated gene rescue a p19 $19^{A R F}$-induced growth arrest in U2-OS cells. Cells were co-infected with the shRNA vectors and LZRS-p19ARF-IRES-zeocin. Fifty thousand cells were seeded and zeocin/puromycin selected. Colonies were stained after two weeks. d, Rescue of a p53-dependent cell-cycle arrest following ionizing radiation. U2-OS cells were co-transfected with the indicated shRNA vectors and H2B-GFP, irradiated (+IR, 10 Gy). GFP-positive cells were analysed by FACS. 
The tumour suppressor p19 $9^{\mathrm{ARF}}$ elicits a p53-dependent proliferation arrest when ectopically expressed ${ }^{13}$. We argued that if the five newly identified genes act in the p53 pathway, their knockdown should confer resistance to $\mathrm{p} 19^{\mathrm{ARF}}$-induced proliferation arrest. To test this, we used a retroviral vector encoding a p19 $9^{\mathrm{ARF}}$-redfluorescent-protein (ARF-RFP) fusion protein to infect human U2-OS osteosarcoma cells. Upon infection of U2-OS cells with this virus, only very few proliferating colonies appeared. The few colonies that appeared did not express the ARF-RFP fusion protein (Fig. 2c; data not shown). By contrast, many ARF-RFP-positive colonies appeared in U2-OS cells in which $p 53$ was suppressed by shRNA (Fig. 2c). Importantly, each of the two independent shRNA vectors against the five newly identified genes allowed proliferation in the presence of ARF-RFP, which is shown by the presence of red fluorescence (Fig. 2c; data not shown). This result provides further support for the notion that the newly identified genes are components of the 553 pathway.

Next, we asked whether suppression of these genes allowed the p53-dependent G1 cell-cycle arrest to be bypassed after exposure to DNA damage. Figure $2 \mathrm{~d}$ shows that exposure of U2-OS cells to ionizing radiation (IR) causes these cells to withdraw from $S$ phase and arrest both in the G1 phase of the cell cycle (a p53-dependent process $^{14}$ ) and in $\mathrm{G} 2 / \mathrm{M}$ (a p53-independent process $\left.{ }^{14}\right)$. As expected, inactivation of p53 by shRNA completely abrogates the G1 fraction after IR, whereas introduction of a shRNA against HDM2 (the human orthologue of the mouse double minute 2 ( $M d m 2$ ) gene), which activates $\mathrm{p} 53$, resulted in a marked increase in the G1 phase of the cell cycle (Fig. 2d). These results demonstrate that arrest in the G1 phase of the cell cycle after IR is determined by the activity of p53. Transfection of knockdown vectors for the five newly identified genes in each case resulted in a cell-cycle distribution identical to the cells expressing the shRNA against p53 (Fig. 2d). Thus, the knockdown of all five genes prevents three different forms of p53-dependent proliferation arrest (Fig. 2b-d).

A major component of the anti-proliferative response of $\mathrm{p} 53$ is the CDK inhibitor $\mathrm{p} 21^{\mathrm{cip} 1}$ (ref. 15), as it is a critical downstream component of the G1 arrest in response to DNA damage ${ }^{16,17}$ and is required for the senescence response of human fibroblasts ${ }^{18}$. To test whether the five newly identified genes affect the expression of known p53 target genes, we stably expressed knockdown vectors for all five genes in U2-OS cells and determined the expression of two p53 target genes, $p 21^{c i p 1}$ and $B A X^{19}$. In addition, p53 protein levels can be used as an indirect read-out for $\mathrm{p} 53$ function, as $\mathrm{p} 53$ protein levels are controlled by the p53 target HDM2 (refs 20, 21). Figure 3a shows that knocking down RPS6KA6, HDAC4, CCT2, KIAA0828 or HTATIP causes a strong reduction in $p 21^{c i p 1}$ messenger RNA and protein levels, but does not affect $\mathrm{p} 53$ protein levels nor the levels of $B A X$ mRNA and protein (Fig. 3a, b). Conversely, overexpression of HTATIP resulted in upregulation of $p 21^{c i p 1}$ expression (Fig. 3c) and further enhanced G1 cell-cycle arrest in response to DNA damage (Fig. 3d). We conclude that knockdown of all five new p53 pathway components leads to a specific downregulation of a subset of $\mathrm{p} 53$ target genes, which includes $p 21^{c i p 1}$.

Next, we tested the significance of the inhibition of $p 21^{\text {cip } 1}$ expression for the phenotypes induced by the newly identified genes. Figure $4 \mathrm{a}$ shows that suppression of $p 21^{c i p 1}$ was nearly as efficient at preventing growth arrest in BJ-TERT-tsLT cells as knockdown of $p 53$ itself. Furthermore, knockdown of $p 21^{\text {cip } 1}$ a

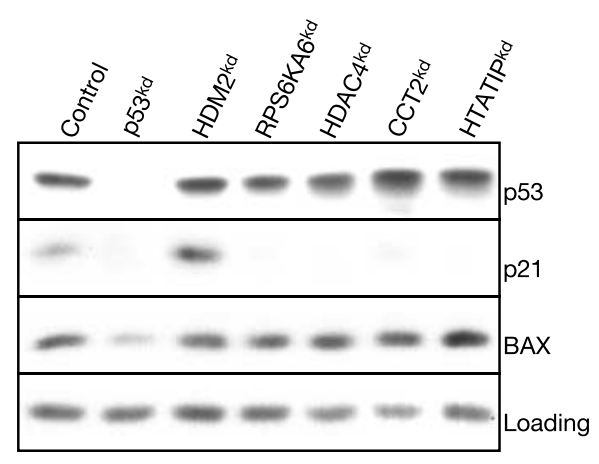

b

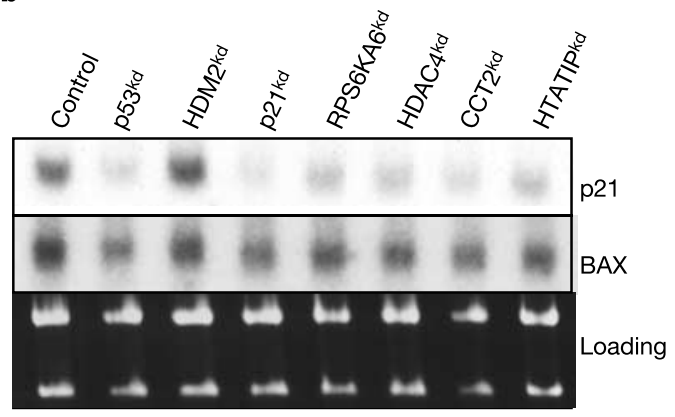

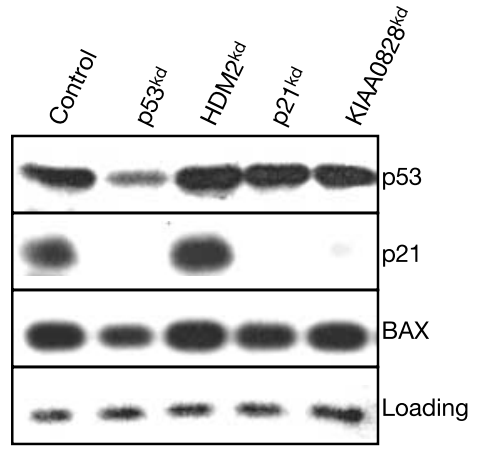

c

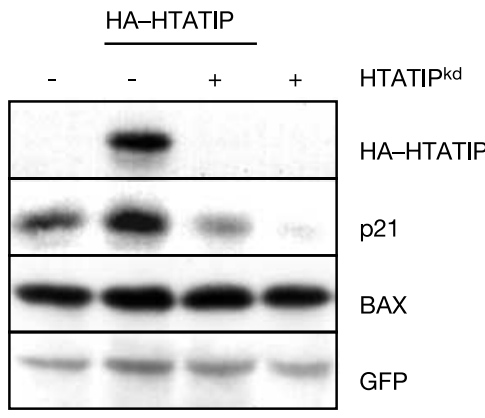

d
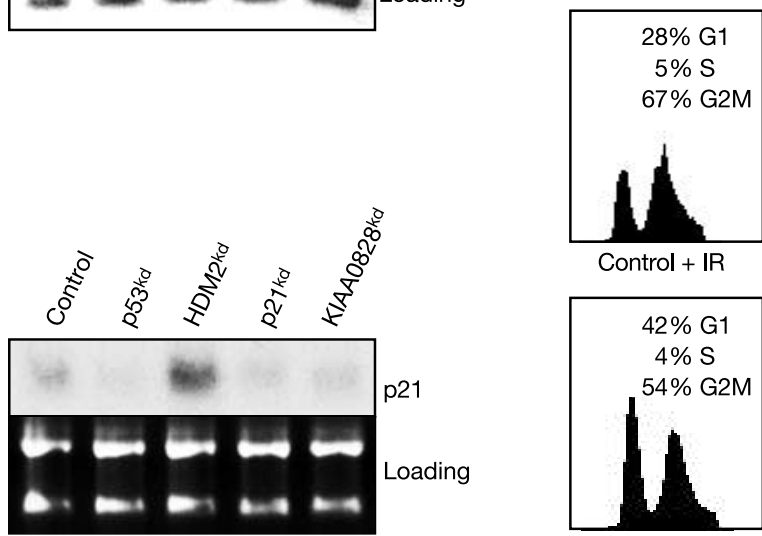

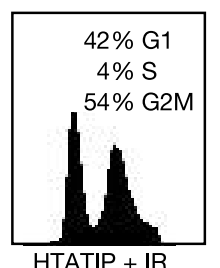

HTATIP + IR

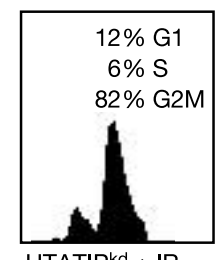

HTATIPkd + IR

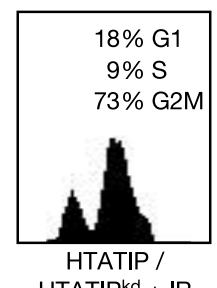

Figure 3 Downregulation of p21 transcription. a, U2-OS cells were stably infected with retroviruses expressing the indicated knockdown constructs. Cell extracts were immunoblotted with antibodies against p53, p21 ${ }^{\mathrm{cip} 1}$ and BAX. b, mRNA levels of p21 and BAX in the stably infected U2-OS cells. As a loading control, RNA was stained with ethidium bromide. c, U2-OS cells were transfected with an expression vector for GFP and
CMV-HA-HTATIP/pRS-HTATIP as indicated. Cell extracts were immunoblotted with antibodies against HA, p21, BAX and GFP. d, Overexpression of HTATIP in U2-OS cells increased the population of cells in the G1 phase of the cell cycle after irradiation $(+\mathbb{R}$, 5 Gy). Co-transfection of a HTATIP shRNA vector reversed the effect of HTATIP overexpression. 
allowed the p53-dependent growth arrest induced by $19^{\mathrm{ARF}}$ (Fig. 4b) and the G1 cell-cycle arrest induced by IR in U2-OS cells (Fig. 4c) to be overridden. This suggests that suppression of $p 21^{c i p 1}$ is a major element in the cellular response to the effect of knocking down the newly identified p53 pathway components. However, we cannot exclude the possibility that knockdown of the newly identified genes has effects on other p53 target genes or p53-independent effects that explain (part of) their knockdown phenotype.

The RNAi screen described above is time consuming in that individual colonies of cells must be isolated and hairpin vectors recovered and tested in second-round selection. We have recently proposed an alternative strategy to rapidly screen complex shRNA vector libraries in a polyclonal format, a technique that we named 'siRNA bar-code screens' ${ }^{22}$. This approach, which was pioneered in yeast $^{23}$, takes advantage of the fact that each hairpin vector contains a unique gene-specific molecular identifier: the 19-mer targeting sequence. This 'molecular bar code' can be used to follow the relative abundance of individual vectors in a large population using DNA microarrays that contain the bar-code oligonucleotides $^{22}$ (Fig. 5a). To test the concept of siRNA bar-code screens, we used a subset of the shRNA library (consisting of 990 different vectors) to infect U2-OS cells. After one day, genomic DNA was isolated from the infected cells, and the shRNA inserts were recovered by PCR with primers flanking the shRNA expression cassette (which includes the 19-mer gene-specific 'bar codes'). The PCR fragments were then labelled with fluorescent dye, and the relative abundance of specific shRNA inserts was determined by hybridization to a DNA microarray containing the 59-mer oligonucleotides used to generate the hairpin vectors (containing the bar- code sequences). Figure 5a shows that the PCR fragments hybridize with high specificity to their complementary 59-mer oligonucleotides on the array.

To further validate the bar-code screening concept, we asked whether we could identify a mouse p53 shRNA vector mixed in a pool of 312 human shRNA vectors in a functional screen. We infected this set of shRNA vectors in conditionally immortalized mouse neuronal cells, which are immortal at $32^{\circ} \mathrm{C}$ but enter into a synchronous p53-dependent growth arrest at $39^{\circ} \mathrm{C}$ (ref. 24). Figure $5 \mathrm{~b}$ shows the design of the experiment and the relative abundance of all the bar codes ten days after shift to $39^{\circ} \mathrm{C}$, which activated the p53-dependent growth arrest ${ }^{24}$. Only the p53 knockdown vectors (indicated in red) were strongly selected in the population, whereas the set of control shRNA vectors was neither positively nor negatively selected. The positive selection of the p53 shRNA vectors occurred over time and only when the p53-dependent growth arrest was invoked $\left(\right.$ at $39^{\circ} \mathrm{C}$ ), and not at $32^{\circ} \mathrm{C}$, which is when p53 is inactive owing to LT binding (Fig. 5d). These data indicate that siRNA bar-code screening can be used to rapidly identify individual shRNA vectors that modulate cellular responses in large populations of vectors.

We identify here five components of the p53 tumour-suppressor pathway through a large-scale RNAi screen in mammalian cells. Inhibition of each of these five genes confers resistance to three different aspects of p53-dependent proliferation arrest, raising the possibility that these genes are tumour-suppressor genes. p53 is a central component of the cellular response to a variety of stress signals, and the genes identified here may be part of the machinery that allow cells to respond to such signals. Our data indicate that the

BJ/ts-LT at $39^{\circ} \mathrm{C}+\mathrm{p} 16^{\mathrm{kd}}$

$\mathbf{a}$

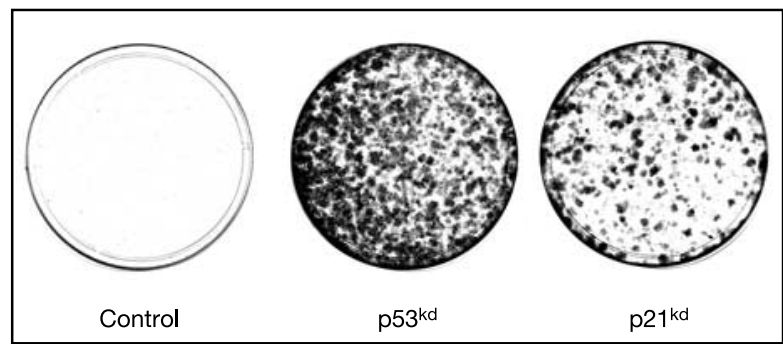

U2-OS / p19ARF-RFP

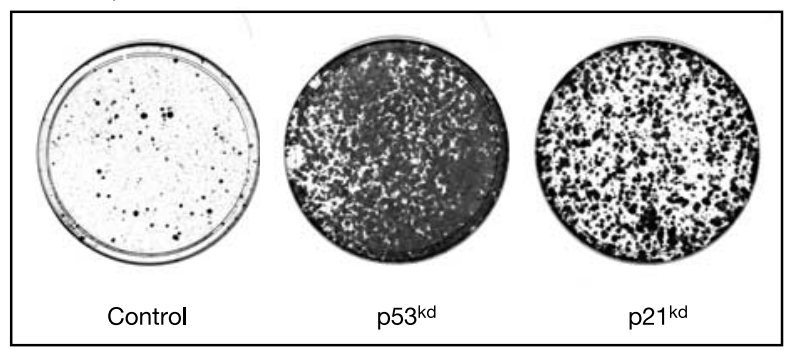

c

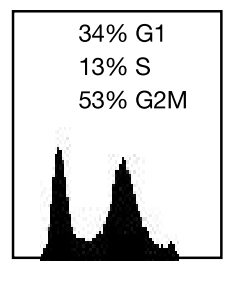

Control + IR

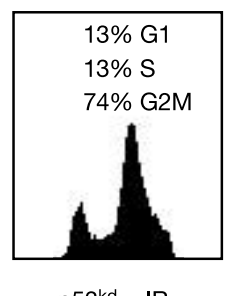

$p 53^{\mathrm{kd}}+\mathrm{IR}$

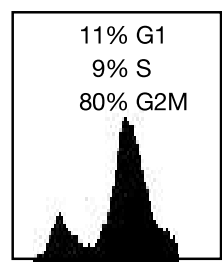

$\mathrm{p} 21^{\mathrm{kd}}+\mathrm{IR}$
Figure 4 Knockdown of $p 21^{\text {cip } 1}$ is sufficient to bypass a p53-dependent arrest. Introduction of p53 and p21 shRNA vectors rescue $\mathbf{a}$, the growth arrest of temperature-shifted BJ-tsLT cells, $\mathbf{b}$, the p19 ${ }^{A R F}$-induced growth arrest in U2-OS cells and $\mathbf{c}$, the p53-induced G1 arrest in U2-OS cells. 

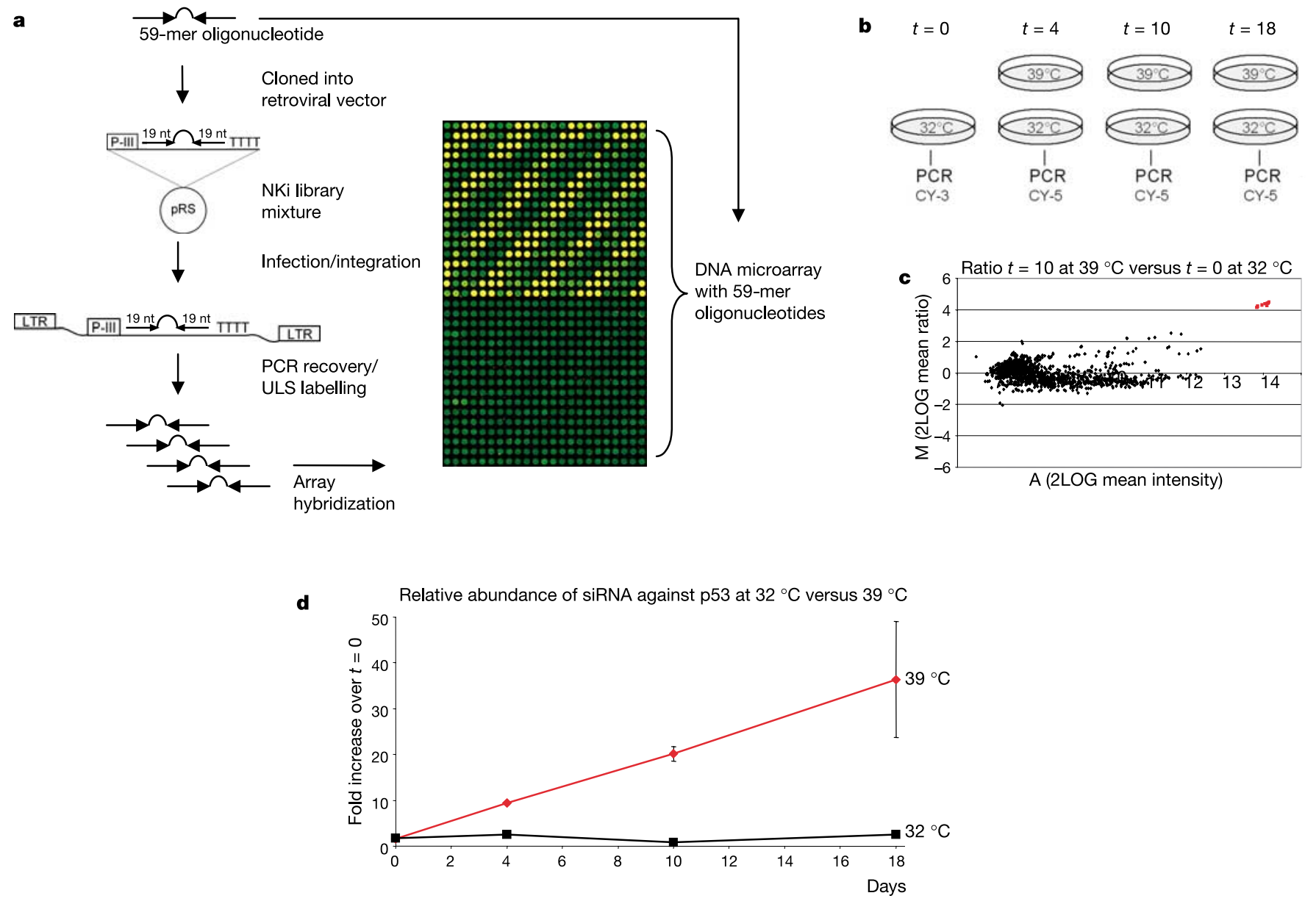

Figure 5 siRNA bar-code screens. a, DNA microarrays containing the 59-mer oligonucleotides present in a selected set of 990 different shRNA vectors were generated. Genomic DNA isolated from cells infected with these 990 shRNA vectors was used for PCR amplification. ULS-labelled bar-code fragments were hybridized on microarrays that consist of oligonucleotides that were present (upper half) or absent (lower half) in the selected set of 990 vectors. $\mathbf{b}$, TsLT immortalized mouse neuronal cells ${ }^{29}$ were infected with a retroviral supernatant containing 312 knockdown vectors directed against human genes and one against murine p53 (ref. 30). Cells were temperature shifted to induce a senescence-like arrest and used for isolation of the bar codes at the indicated time points. c. Comparative hybridization of bar-code fragments isolated from the experiment described in $\mathbf{b}$ at $t=0$ with bar-code fragments isolated ten days after the temperature shift. Red spots indicate the relative abundance of the p53 shRNA. Note that only the relative abundance of the p53 knockdown vector has increased. d, Analysis of the relative abundance of $\mathrm{p} 53$ shRNAs in tsLT-immortalized mouse neuronal cells recovered from cells grown at $32^{\circ} \mathrm{C}$, when endogenous $p 53$ is inactivated by the tsLT antigen, or at $39^{\circ} \mathrm{C}$, when p53 is activated owing to loss of tsLT antigen expression. newly identified genes have a role, either directly or indirectly, in modulating the activity of $\mathrm{p} 53$ on the $p 21^{\text {cip } 1}$ promoter. We did not identify the shRNA for $p 21^{c i p 1}$ in the genetic screen, even though it was present in the RNAi library and active in $p 21^{\text {cip } 1}$ suppression. This indicates that the first screen was not saturating and that additional p53 pathway components may be identified from this library.

Our data highlight the power of large-scale RNAi screens in mammalian cells. A potentially useful application of siRNA barcode screens is in the identification of synthetic lethal interactions (a combination of two non-lethal mutations that together result in cell death). The identification of such genetic interactions in mammalian cells may facilitate the development of new and more specific classes of anticancer drugs.

\section{Methods}

\section{Materials, antibodies and vectors}

IR was performed with a $2 \times 415 \mathrm{Ci}^{137} \mathrm{Cs}$ source. Antibodies against $\mathrm{p} 21(\mathrm{C}-19)$, BAX (N20), green fluorescent protein (GFP; FL), haemagglutinin (HA; Y-11) and CDK4 (C-22) were obtained from Santa Cruz, anti-Flag M2 from Sigma and anti-p53 (ab-7) from Oncogene Research Products. HA-tagged HTATIP was PCR amplified and cloned in pRC/ CMV (Invitrogen). Flag-tagged RPS6KA6, CCT2 and KIAA0828 were generated by PCR amplification and cloning into pcDNA3.1/HygroFlag. pRS, pRS-p16 ${ }^{\mathrm{kd}}$, LZRS-p19 ${ }^{\mathrm{ARF}}$ RFP-IRES-zeocin, Flag-HDAC4 and H2B-GFP have been described previously ${ }^{9,25,26}$. pEGFP-N1 was obtained from Clontech. For the generation of knockdown vectors for $\mathrm{p} 53$, p21 and HDM2, the following 19-nucleotide sequences were used: p53, 5' -

CTACATGTGTAACAGTTCC-3'; p21, 5' -GACCATGTGGACCTGTCAC-3' ; and HDM2, $5^{\prime}$-GATGATGAGGTATATCAAG-3 ${ }^{\prime}$. Control transfections were performed with a mixture of non-functional hairpins.

\section{Oligonucleotide design and library construction}

A representative mRNA sequence was selected for each target from UniGene (http:// www.ncbi.nlm.nih.gov/entrez/query.fcgi? $\mathrm{db}=$ unigene). Sequences were masked to remove repetitive sequences using RepeatMasker (http://ftp.genome.washington.edu/cgibin/RepeatMasker), and vector contamination was masked by searching with NCBI BLAST (http://www.ncbi.nlm.nih.gov/BLAST/) against UniVec (http://

www.ncbi.nih.gov/VecScreen/UniVec.html). Three unique 19-mers for each target were selected, where possible, (1) to contain no stretches of four or more consecutive T or A residues (to avoid premature pol III transcription termination signals); (2) to have $30-70 \%$ overall GC content; (3) to lie within the coding sequence of the target gene; (4) to begin with a $\mathrm{G}$ or $\mathrm{C}$ residue (consistent with recently established rules for strand bias ${ }^{27}$ ); (5) to begin after an AA dimer in the $5^{\prime}$ flanking sequence; (6) to end just prior to a TT, TG or GT doublet in the $3^{\prime}$ flanking sequence; (7) to not contain XhoI or EcoRI restrictionenzyme sites to facilitate subsequent shuttling of the knockdown cassette into other vector backbones; (8) to share minimal sequence identity with other genes; (9) to target all transcript variants represented by RefSeq mRNAs (http://www.ncbi.nlm.nih.gov/ RefSeq/); and (10) to not overlap with other 19-mers selected from the same target sequence.

For cloning, pairs of complementary oligonucleotides were annealed and ligated into 
HindIII/BglII-digested pRS in 96-well plates. Ligation reactions were transformed into competent DH5 $\alpha$ bacteria. Bacterial cultures were grown overnight and plasmid DNA was isolated. All manipulations were performed using a Multimek robot.

Additional information about the NKi RNAi library can be found at http:// screeninc.nki.nl/.

\section{Cell culture, transfection and retroviral infection}

BJ fibroblasts were cultured in a 4:1 mixture of DMEM:M199 supplemented with 15\% heat-inactivated fetal calf serum. U2-OS and Phoenix cells were cultured in DMEM supplemented with $10 \%$ heat-inactivated fetal calf serum. Transfections were performed with the calcium phosphate precipitation technique. Ecotropic retoviral supernatants were produced by transfection of Phoenix packaging cells. Viral supernatants were filtered through a $0.45 \mu \mathrm{m}$ filter, and infections were performed in the presence of $4 \mu \mathrm{g} \mathrm{ml}^{-1}$ polybrene (Sigma). Drug selections in U2-OS cells were performed with $2 \mu \mathrm{g} \mathrm{ml}$ puromycin and $200 \mu \mathrm{g} \mathrm{ml}^{-1}$ zeocin (Invitrogen).

\section{Recovery of shRNA inserts}

Genomic DNA was isolated from expanded colonies using DNAzol (Life Technologies). PCR amplification of the shRNA inserts was performed with Expand Long Template PCR system (Roche) and the use of pRS-fw primer: $5^{\prime}$-CCCTTGAACCTCCTCGTTCGACC- 3 and pRS-rev primer: $5^{\prime}$-GAGACGTGCTACTTCCATTTGTC- $3^{\prime}$. Products were digested with EcoRI/XhoI and recloned into pRS. Hairpins were sequenced with Big Dye Terminator (Perkin Elmer) using pRS-seq primer: 5' -GCTGACGTCATCAACCCGCT-3'

\section{Cell-cycle analysis}

For fluorescence-activated cell sorting (FACS) analysis, U2-OS cells were co-transfected with H2B-GFP to select for transfected cells. Forty-eight hours after transfection, cells were treated with IR ( 5 or $10 \mathrm{~Gy}$ ). Twenty-four hours after IR, the cells were washed and fixed in $70 \%$ ethanol at $4{ }^{\circ} \mathrm{C}$. Before FACS analysis, cells were washed with PHN (PBS, $20 \mathrm{mM}$ HEPES, $0.5 \% \mathrm{NP} 40$ ) and incubated with $10 \mu \mathrm{g} \mathrm{ml}^{-1}$ propidium iodide and $250 \mu \mathrm{g} \mathrm{ml}^{-1}$ RNase A. In each assay, 8,000 GFP-positive cells were collected by FACScan and analysed using the CellQuest program (Becton Dickinson).

\section{Western and northern blotting}

For western blots, puromycin-selected cells were lysed in RIPA buffer $(50 \mathrm{mM}$ Tris $\mathrm{pH} 8$ $150 \mathrm{mM} \mathrm{NaCl} ; 1 \% \mathrm{NP} 40 ; 0.5 \%$ DOC; $0.1 \% \mathrm{SDS}$ ). Thirty micrograms of protein was separated on $8-12 \%$ SDS-polyacrylamide gel electrophoresis and transferred to polyvinylidine difluoride membranes (Millipore). Western blots were probed with antibodies. For northern analysis, $15 \mu \mathrm{g}$ total RNA was loaded on a $1 \%$ agarose gel and blotted onto Hybond $\mathrm{N}+$ (Amersham). ${ }^{32} \mathrm{P}$-labelled probes for $\mathrm{p} 21$ and BAX were generated with PCR labelling.

\section{siRNA bar-code screens}

The shRNA inserts were amplified from genomic DNA by PCR using the primers pRS-fw primer $5^{\prime}$-CCCTTGAACCTCCTCGTTCGACC-3' and pRS8-rev primer $5^{\prime}$

TAAAGCGCATGCTCCAGACT- $3^{\prime}$. PCR products were labelled with cyanine-3 or cyanine-5 fluorescent groups using the Universal Linkage System (ULS; Kreatech Biotechnology) and purified over a KreaPure (Kreatech Biotechnology) spin column as described previously ${ }^{28}$. PCR products from two samples were combined and hybridized to oligonucleotide arrays in $40 \mu \mathrm{l}$ of $25 \%$ formamide, $5 \times$ SCC, $0.01 \%$ SDS (containing poly $\mathrm{d}(\mathrm{A})$, yeast transfer RNA and COT-1 DNA). Samples were heated to $100^{\circ} \mathrm{C}$ for $1 \mathrm{~min}$ and applied to the array. Samples were hybridized for $18 \mathrm{~h}$ at $42^{\circ} \mathrm{C}$, washed and scanned using an Agilent microarray scanner. Quantification of the resulting fluorescent images was performed with Imagene 5.6 (BioDiscovery); the local background was subtracted and the data normalized and ${ }^{2} \log$ transformed. Additional information on bar-code screens can be found at http://screeninc.nki.nl/.

Received 11 November 2003; accepted 26 January 2004; doi:10.1038/nature02371.

1. Lum, L. et al. Identification of Hedgehog pathway components by RNAi in Drosophila cultured cells Science 299, 2039-2045 (2003).

2. Kamath, R. S. et al. Systematic functional analysis of the Caenorhabditis elegans genome using RNAi. Nature 421, 231-237 (2003).

3. Ashrafi, K. et al. Genome-wide RNAi analysis of Caenorhabditis elegans fat regulatory genes. Nature 421, 268-272 (2003)

4. Brummelkamp, T. R., Bernards, R. \& Agami, R. A system for stable expression of short interfering RNAs in mammalian cells. Science 296, 550-553 (2002).

5. Paddison, P. J., Caudy, A. A., Bernstein, E., Hannon, G. J. \& Conklin, D. S. Short hairpin RNAs (shRNAs) induce sequence-specific silencing in mammalian cells. Genes Dev. 16, 948-958 (2002).

6. Hannon, G. J. RNA interference. Nature 418, 244-251 (2002).

7. Elbashir, S. M. et al. Duplexes of 21-nucleotide RNAs mediate RNA interference in cultured mammalian cells. Nature 411, 494-498 (2001).

8. Sherr, C. J. The ink4a/ARF network in tumour suppression. Nature Rev. Mol. Cell Biol. 2, 731-737 (2001).

9. Brummelkamp, T., Bernards, R. \& Agami, R. Stable suppression of tumorigenicity by virus-mediated RNA interference. Cancer Cell 2, 243-247 (2002).

10. Brummelkamp, T. R., Nijman, S. M., Dirac, A. M. \& Bernards, R. Loss of the cylindromatosis tumour suppressor inhibits apoptosis by activating NF-kB. Nature 424, 797-801 (2003).

11. Semizarov, D. et al. Specificity of short interfering RNA determined through gene expression signatures. Proc. Natl Acad. Sci. USA 100, 6347-6352 (2003).

12. Jackson, A. L. et al. Expression profiling reveals off-target gene regulation by RNAi. Nature Biotechnol. 21, 635-637 (2003)

13. Kamijo, T. et al. Tumor suppression at the mouse INK4a locus mediated by the alternative reading frame product p19ARF. Cell 91, 649-659 (1997).
14. Lakin, N. D. \& Jackson, S. P. Regulation of p53 in response to DNA damage. Oncogene 18, 7644-7655 (1999)

15. El-Deiry, W. S. et al. WAF1, a potential mediator of p53 tumor suppression. Cell 75, 817-825 (1993)

16. Brugarolas, J. et al. Radiation-induced cell cycle arrest compromised by p21 deficiency. Nature 377, 552-557 (1995).

17. Waldman, T., Kinzler, K. W. \& Vogelstein, B. p21 is necessary for the p53-mediated G1 arrest in human cancer cells. Cancer Res. 55, 5187-5190 (1995).

18. Brown, J. P., Wei, W. \& Sedivy, J. M. Bypass of senescence after disruption of p21CIP1/WAF1 gene in normal diploid human fibroblasts. Science 277, 831-834 (1997).

19. Miyashita, T. \& Reed, J. C. Tumor suppressor p53 is a direct transcriptional activator of the human bax gene. Cell 80, 293-299 (1995).

20. Barak, Y., Juven, T., Haffner, R. \& Oren, M. mdm2 expression is induced by wild type p53 activity. EMBO J. 12, 461-468 (1993).

21. Kubbutat, M. H., Jones, S. N. \& Vousden, K. H. Regulation of p53 stability by Mdm2. Nature 387, 299-303 (1997).

22. Brummelkamp, T. R. \& Bernards, R. New tools for functional mammalian cancer genetics. Nature Rev. Cancer 3, 781-789 (2003).

23. Shoemaker, D. D., Lashkari, D. A., Morris, D., Mittmann, M. \& Davis, R. W. Quantitative phenotypic analysis of yeast deletion mutants using a highly parallel molecular bar-coding strategy. Nature Genet. 14, 450-456 (1996).

24. Brummelkamp, T. R. et al. TBX-3, the gene mutated in Ulnar-Mammary Syndrome, is a negative regulator of p19ARF and inhibits senescence. J. Biol. Chem. 277, 6567-6572 (2002).

25. Kanda, T., Sullivan, K. F. \& Wahl, G. M. Histone-GFP fusion protein enables sensitive analysis of chromosome dynamics in living mammalian cells. Curr. Biol. 8, 377-385 (1998).

26. Wang, A. H. et al. HDAC4, a human histone deacetylase related to yeast $\mathrm{HDAl}$, is a transcriptional corepressor. Mol. Cell. Biol. 19, 7816-7827 (1999).

27. Schwarz, D. S. et al. Asymmetry in the assembly of the RNAi enzyme complex. Cell 115, 199-208 (2003)

28. Heetebrij, R. J. et al. Platinum(II)-based coordination compounds as nucleic acid labeling reagents: synthesis, reactivity, and applications in hybridization assays. Chembiochem 4, 573-583 (2003).

29. Trettel, F. et al. Dominant phenotypes produced by the HD mutation in STHdh(Q111) striatal cells. Hum. Mol. Genet. 9, 2799-2809 (2000).

30. Dirac, A. M. \& Bernards, R. Reversal of senescence in mouse fibroblasts through lentiviral suppression of p53. J. Biol. Chem. 278, 11731-11734 (2003).

Supplementary Information accompanies the paper on www.nature.com/nature.

Acknowledgements We thank S. Friend and J. Downward for their support of this project, M. Voorhoeve, Z. Wu, X.-j. Yang, H. Yntema and Kreatech Biotechnology for reagents, the NKI microarray facility group for assistance, A. Dirac and S. Nijman for technical help, and members of the Bernards laboratory for discussions. This work was supported by grants from the Netherlands Genomics Initiative/Netherlands Organization for Scientific Research (NWO), Cancer Research UK (CRUK), the Centre for Biomedical Genetics (CBG), the Dutch Cancer Society (KWF) and Utrecht University (ABC cluster).

Competing interests statement The authors declare that they have no competing financial interests.

Correspondence and requests for materials should be addressed to R.L.B. (r.beijersbergen@nki.nl) or R.B. (r.bernards@nki.nl).

\section{Functional interactions between receptors in bacterial chemotaxis}

\section{Victor Sourjik $*$ \&oward C. Berg}

${ }^{1}$ Department of Molecular and Cellular Biology, Harvard University, 16 Divinity Avenue, Cambridge, Massachusetts 02138, and the Rowland Institute at Harvard, 100 Edwin H. Land Boulevard, Cambridge, Massachusetts 02142, USA

* Present address: ZMBH, University of Heidelberg, Im Neuenheimer Feld 282, D-69120, Germany

Bacterial chemotaxis is a model system for signal transduction, noted for its relative simplicity, high sensitivity, wide dynamic range and robustness. Changes in ligand concentrations are sensed by a protein assembly consisting of transmembrane receptors, a coupling protein $(\mathrm{CheW})$ and a histidine kinase $(\mathrm{CheA})^{1-4}$. In Escherichia coli, these components are organized at the cell poles in tight clusters that contain several thousand copies of each protein ${ }^{1,4-6}$. Here we studied the effects of variation 